\title{
Improved description of the Roper resonance in a constituent quark model
}

\author{
Fl. Stancu and P. Stassart \\ Université de Liege, Institut de Physique B.5, Sart Tilman, 4000 Liege 1, Belgium \\ (Received 1 August 1989)
}

\begin{abstract}
Within a semirelativistic constituent quark model based on linear confinement, we propose a new radial form for the main component of the Roper resonance. This lowers its position by about 100 $\mathrm{MeV}$ compared to previous studies, where the radial excitation was of harmonic-oscillator type, and brings it much closer to the experiment.
\end{abstract}

Our framework is a constituent quark model ${ }^{1-6}$ which has been previously applied to the calculation of the baryon spectrum ${ }^{2}$ and of the radiative ${ }^{3}$ and strong decay $^{4-6}$ of $N$ and $\Delta$ resonances into $N+\pi$. An important drawback of the model was the position of the Roper resonance $P_{11}(1440)$ which appeared at about $150 \mathrm{MeV}$ above its experimental value. In the present work we propose an improvement of this situation.

The Hamiltonian of the model contains a spinindependent $H_{0}$ (color-electric) and a spin-dependent $H^{\text {hyp }}$ (color-magnetic) part as described in Ref. 2. Its spin-independent part has the form ${ }^{1}$

$$
H_{0}=\sum_{i}\left(p_{i}^{2}+m^{2}\right)^{1 / 2}+V\left(r_{1}, r_{2}, r_{3}\right)+E_{0}^{B}
$$

with

$$
\begin{aligned}
V\left(r_{1}, r_{2}, r_{3}\right)= & \left.\frac{1}{2} \sum_{i<j} \mid-\frac{4}{3} \frac{\alpha_{s}}{r_{i j}}+\sqrt{\sigma} r_{i j}\right) \\
& +\sqrt{\sigma}\left(\sum_{i} r_{i 4}-\frac{1}{2} \sum_{i<j} r_{i j}\right)
\end{aligned}
$$

and

$$
E_{0}^{B}=-1265 \mathrm{MeV} \text {. }
$$

$E_{0}^{B}$ is a constant that can be determined from data, $m$ is the $u$ or $d$ constituent quark mass, $\alpha_{s}$ the strong coupling constant, $\sqrt{\sigma}$ the string tension, $r_{i j}$ the interquark distance, and $r_{i 4}$ the distance between the quark $i$ and a point $\mathbf{r}_{4}$ where the flux tubes meet at $120^{\circ}$. For angles larger than $120^{\circ}$ this point becomes identical to one of the quark positions $r_{i}$.

For the ground state (g.s.) of $H_{0}$ we adopt the variational solution of Ref. 1:

$$
\psi_{0}=F_{123} \sum_{i<j} f\left(r_{i j}\right) \text {, }
$$

where $f\left(r_{i j}\right)$ and $F_{123}$ are two- and three-body correlation functions associated with the first and second terms of (2), respectively. Their analytic form with variational parameters can be found in Ref. 1 .

In the following, instead of $r_{i j}$ we shall use the Jacobi coordinates

$$
\boldsymbol{\rho}=\frac{1}{\sqrt{2}}\left(\mathbf{r}_{1}-\mathbf{r}_{2}\right), \quad \lambda=\frac{1}{\sqrt{6}}\left(\mathbf{r}_{1}+\mathbf{r}_{2}-2 \mathbf{r}_{3}\right) .
$$

The hyperfine interaction $H^{\text {hyp }}$ has a spin-spin part $V_{\text {SS }}$ and a tensor part $V_{T}$

$$
\begin{aligned}
H^{\mathrm{hyp}}= & V_{\mathrm{SS}}+V_{T}, \\
V_{\mathrm{SS}}= & \frac{4 \sqrt{2} \pi \alpha_{s}}{9 m^{2}} \frac{1}{\left(2 \pi \Lambda^{2}\right)^{3 / 2}} e^{-\rho^{2} / 2 \Lambda^{2}} \mathbf{S}_{1} \cdot \mathbf{S}_{2}, \\
V_{T}= & \frac{\alpha_{s}}{\sqrt{2} m^{2} \rho^{3}}\left[\operatorname{erf}\left(\frac{\rho}{\sqrt{2} \Lambda}\right)\right. \\
& \left.\quad-\frac{\sqrt{2}}{3 \sqrt{\pi}} \frac{\rho^{3}}{\Lambda^{3}}\left(1+3 \frac{\Lambda^{2}}{\rho^{2}}\right) e^{-\rho^{2} / 2 \Lambda^{2}}\right] \\
& \times\left(\frac{1}{\rho^{2}} \mathbf{S}_{1} \cdot \rho \mathbf{S}_{2} \cdot \rho-\frac{1}{3} \mathbf{S}_{1} \cdot \mathbf{S}_{2}\right) .
\end{aligned}
$$

It contains two parameters: the mass $m$ and the finite size $\Lambda$ of the quark. In order to make a comparison with previous results we use the so-called set II of Ref. 4:

$$
m=324 \mathrm{MeV}, \quad \Lambda=0.09 \mathrm{fm}
$$

which gave a good overall description of the spectrum except for the Roper resonance.

TABLE I. The constants $N_{0}^{\prime}$ and $\alpha$ and the energy difference between the Roper resonance and the ground state corresponding to various values of $k$ in Eq. (10).

\begin{tabular}{lcccc}
\hline \multicolumn{1}{c}{$k$} & 1 & 2 & 3 & 4 \\
\hline$N_{0}^{\prime}$ & 0.030 & 0.058 & 0.087 & 0.116 \\
$\alpha$ & 1.317 & 1.682 & 2.091 & 2.533 \\
$E_{\text {Roper }}-E_{N}$ & 564 & 581 & 614 & 666 \\
\hline \hline
\end{tabular}


The hyperfine interaction is diagonalized in a truncated space based on the usual ${ }^{7} \mathrm{SU}(6)$ flavor $X$ spin scheme. As in Refs. 1-6 it contains one unit of radial excitation and up to two units of orbital excitation.

The space part of an excited state has the general form

$$
\psi_{n}(\rho, \lambda)=\phi_{n}(\rho, \lambda) \psi_{0}
$$

where $\phi_{n}(\rho, \lambda)$ are chosen such as to ensure orthogonality of $\psi_{n}$. In the present work we propose to describe one unit of radial excitation by

$$
\phi_{0}^{\prime}=\left(N_{0}^{\prime}\right)^{-1}\left[1-\alpha\left(\rho^{2}+\lambda^{2}\right)^{k / 4}\right] .
$$

For $k=1, \ldots, 4$ the normalization factor $N_{0}^{\prime}$ and $\alpha$ determined from orthogonality are given in Table I. The value $k=4$ corresponds to the harmonic-oscillator case which was a natural first step in Refs. 1-6. Equation (10) with $k<4$ has been inspired by the asymptotic form of the Airy function ${ }^{8}$

$$
\operatorname{Ai}(z) \sim \frac{1}{2} \pi^{-1 / 2} z^{-1 / 4} \exp \left(-\frac{2}{3} z^{3 / 2}\right)
$$

to which the solution of a linear potential tends at large distances.

We anticipate by mentioning that we found a convergent lowering of the mass difference between the Roper resonance and the ground state going from $k=4$ to $k=1$ (Table I). In the following we shall present results only for $k=1$ and compare them to $k=4$ (Refs. 2-6). In general, whatever is the choice of $\phi_{n}$ in (9), the diagonalization of the Hamiltonian in a space of orthogonal functions $\psi_{n}$ would give upper bounds as stated by a theorem proved in Ref. 9.

In calculating the mass spectrum a first step is to find the kinetic contribution to $H_{0}$. This step follows closely the prescription of Ref. 1 . In terms of the relative coordinates $(5)$ one can introduce the probability density $P(p)$ of a quark in the state $\psi$ to have a momentum $p$ :

$$
\begin{gathered}
P(p)=\frac{1}{N} \int d^{3} \rho d^{3} \lambda d^{3} \lambda^{\prime} \psi^{*}(\rho, \lambda) \psi\left(\rho, \lambda^{\prime}\right) \\
\times \frac{\sin \left(\frac{3}{2}\right)^{1 / 2} p\left|\lambda-\lambda^{\prime}\right|}{\left(\frac{3}{2}\right)^{1 / 2} p\left|\lambda-\lambda^{\prime}\right|},
\end{gathered}
$$

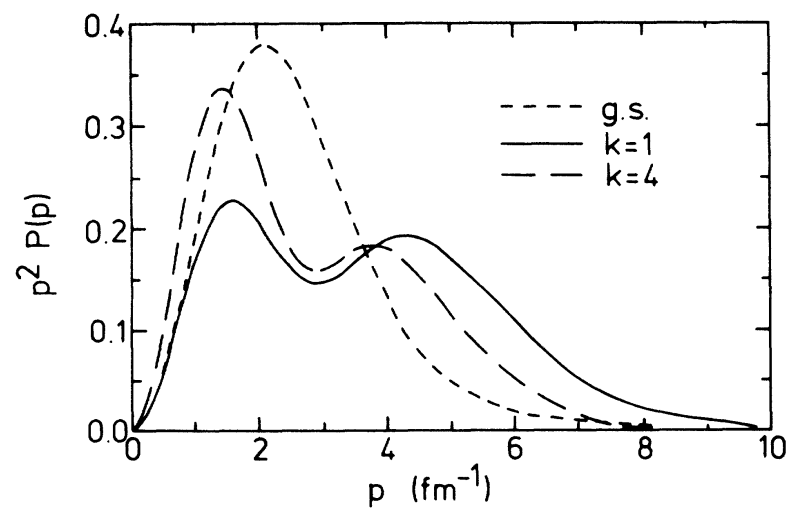

FIG. 1. Momentum distribution of three quarks in the ground state $\psi_{0}$ and radial excitations $\psi_{0}^{\prime}$ with $k=1$ and $k=4$ (harmonic-oscillator type).
TABLE II. Root-mean-square radius ( $\mathrm{fm}$ ) of a three-quark system in the state $\psi_{0}$ and $\psi_{0}^{\prime}$ with $k=1$ and $k=4$ (no hyperfine interaction).

\begin{tabular}{lccc}
\hline \hline State & $\psi_{0}$ & $\psi_{0}^{\prime}(k=1)$ & $\psi_{0}^{\prime}(k=4)$ \\
\hline$\left\langle r^{2}\right\rangle^{1 / 2}$ & 0.36 & 0.42 & 0.52 \\
\hline
\end{tabular}

where $N$ is determined from the normalization condition

$$
\int P(p) p^{2} d p=1 \text {. }
$$

The kinetic energy is then

$$
T=\int P(p)\left(m^{2}+p^{2}\right)^{1 / 2} p^{2} d p .
$$

The nine-dimensional integral (12) has been evaluated by the Monte Carlo method. The quantity $p^{2} P$ is plotted in Fig. 1 as a function of $p$ for $\psi_{0}$ of Eq. (4) and for $\psi_{0}^{\prime}=\phi_{0}^{\prime} \psi_{0}$ with $k=1$ and $k=4$ in Eq. (10), respectively. At any $p$ the statistical Monte Carlo error has been kept below 2\% of the peak value of each curve. In all cases the upper limit of the integral (14) is large enough to make the contribution of the tail of $p^{2} P$ negligible. The largest resulting statistical error on $T$ is $30 \mathrm{MeV}$ for $k=1$, where the integration extends over a wider interval. In fact from Fig. 1 one can see that the momentum distribution of the two excited $\psi_{0}^{\prime}$ states have similar shapes but it is more extended towards larger $p$ when $k=1$. This has a consequence on the radii as seen in Table II. These radii are calculated from $\psi_{0}$ or $\psi_{0}^{\prime}$, i.e., before including the hyperfine splitting. The expectation value of the kinetic and potential parts of $H_{0}$ are displayed in various columns of Table III for the ground state and the two $\psi_{0}^{\prime}$ states under consideration. The sum of these contributions reproduces nearly identically the result of Ref. 1 for $\psi_{0}$ and the harmonic-oscillator type $\psi_{0}^{\prime}$.

The results of the diagonalization of the hyperfine interaction (6) and (7) for the sector $J^{\pi}=\frac{1}{2}^{+}$are presented in Table IV. One can see that the mass of the Roper resonance is now only $564 \mathrm{MeV}$ above the ground state while in Ref. 3 it was $666 \mathrm{MeV}$. Table III shows that the expectation value of $H_{0}$ is about $35 \mathrm{MeV}$ higher for $k=1$ than for $k=4$. The sizable lowering of the Roper resonance for $k=1$ is therefore due to the hyperfine interaction. The whole spectrum can be shifted by slightly readjusting $E_{0}^{B}$ in order to reproduce the nucleon mass. For completeness we present in Table $I$ the mass difference between the Roper resonance and the corresponding ground state for all $k=1, \ldots, 4$ initially considered in Eq. (10).

TABLE III. Detailed contributions (in $\mathrm{MeV}$ ) to the expectation value of $H_{0}$ of Eqs. (1)-(3).

\begin{tabular}{lcrcr}
\hline \hline State & $\left\langle\left(m^{2}+p^{2}\right)^{1 / 2}\right\rangle\left\langle-\frac{2}{3} \frac{\alpha_{s}}{r_{i j}}\right\rangle\left\langle\frac{1}{2} \sqrt{\sigma} r_{i j}\right\rangle\left\langle\sqrt{\sigma}\left(r_{i 4}-\frac{1}{2} r_{i j}\right)\right\rangle$ \\
\hline$\psi_{0}$ & 1832 & -344 & 853 & 81 \\
$\psi_{0}^{\prime}(k=4)$ & 1974 & -291 & 1188 & 108 \\
$\psi_{0}^{\prime}(k=1)$ & 2418 & -408 & 919 & 85 \\
\hline
\end{tabular}


TABLE IV. $J^{\pi}=\frac{1}{2}^{+}$nonstrange baryons. Masses $(\mathrm{MeV})$ and mixing angles resulting from the hyperfine interaction $(6)-(8)$.

\begin{tabular}{lrrrrrr}
\hline \hline Symmetry & Mass & \multicolumn{5}{c}{ Mixing angles } \\
\hline${ }^{2} N\left(56,0^{+}\right)$ & 921 & 0.945 & 0.281 & -0.163 & -0.033 & 0.002 \\
${ }^{2} N\left(56,0^{+}\right)$ & 1485 & 0.261 & -0.956 & -0.135 & -0.001 & $5 \times 10^{-5}$ \\
${ }^{2} N\left(70,0^{+}\right)$ & 1796 & -0.175 & 0.083 & -0.934 & 0.293 & -0.067 \\
${ }^{4} N\left(70,2^{+}\right)$ & 1930 & 0.082 & -0.017 & 0.276 & 0.824 & -0.488 \\
${ }^{2} N\left(20,1^{+}\right)$ & 2042 & -0.031 & 0.004 & -0.084 & -0.484 & -0.870 \\
\hline \hline
\end{tabular}

The mixing angles in the sector $J^{\pi}=\frac{1}{2}^{+}$have some changes compared to those of Ref. 4. The most important is that for $k=1$ the mixing angles between ${ }^{2} N\left(56,0^{+}\right)$and ${ }^{2} N\left(566^{\prime}, 0^{+}\right)$configurations increase by about $65 \%$ with respect to $k=4$. In order to test the new composition of the states we have calculated the radiative decays following the method of Ref. 3 and the strong decays into $N+\pi$ using the breaking flux-tube mechanism of Ref. 6. The results are given in Table V. For each resonance the first row reproduces the results of previous calculations $(k=4)$, the second the present results $(k=1)$, and the third the data corresponding to the two experimentally known $P_{11}$ resonances. ${ }^{10}$

For the strong decays the quantity to be discussed is the square root of the decay width $\Gamma_{N \pi}^{1 / 2}$. For $P_{11}(1440)$ the two theoretical values of $\Gamma_{N \pi}^{1 / 2}$ are very close in magnitude and within the experimental range. This shows that the width is dominated by the effects of the nonlocality in the pion emission operator ${ }^{5,6,11}$ and not much influenced by the form of the radial part of the wave function. For $P_{11}(1710)$ the $k=1$ excitation produces a decrease in $\Gamma_{N \pi}^{1 / 2}$ but not much out of the experimental range.

In the table we also reproduce the sign of the calculated $A_{1 / 2 N \pi}$ amplitudes. This is used in establishing the sign of the helicity amplitudes of the radiative decays as explained in Ref. 3. For the Roper resonance the sign is the same for $k=4$ and $k=1$ and gives the correct sign for the photodecay amplitudes. For the $P_{11}(1710)$ resonance and theoretical signs are opposite. It means that both the amplitude and its sign are sensitive to the structure of the state, i.e., changing the basic ansatz for the radial excitation and hence the mixing angles, leads to changes in the decay properties of the $P_{11}(1710)$ resonances. Unfortunately no definite conclusion can be drawn about the correctness of the sign as long as the experimental errors remain large.

Concerning the magnitude for $k=1$, the helicity amplitudes $A_{1 / 2}^{p}$ and $A_{1 / 2}^{n}$ of the Roper resonance decrease by about $30 \%$ with respect to $k=4$ (Ref. 3) and further increase the disagreement with the experiment. The ratio $A_{1 / 2}^{p} / A_{1 / 2}^{n}$ does not change appreciably. It is about -1.6 and lies within the experimental error range. This is a test for the $\mathrm{SU}(6)$ multiplet composition of the state. If both the Roper resonance and the ground state were described by the $(56)+\left(56^{\prime}\right)$ representation only, then the theory would give $A_{1 / 2}^{p} / A_{1 / 2}^{n}=-\frac{3}{2}$ (see Appendix $\mathrm{C}$ of Ref. 3). Since both models predict a dominant contribution of this representation $(97 \%$ for the ground state and 98\% for the Roper resonance) the ratio remains close to -1.5 . For $P_{11}(1710)$ the magnitude is not much affected by the form of the radial excitation.

The photodecay amplitudes have been calculated using a nonrelativistic quark-photon interaction. Its simplicity has been convenient in a first estimate for a large number of resonances. ${ }^{3}$ But contributions of other effects such as relativistic or nonsingular quark transition effects ${ }^{12}$ can produce changes that may be important.

Because of the truncation of the space the negativeparity spectrum is not affected by the ansatz (10) because these states contain only orbital $(L=1)$ excitations. It would be interesting to see what modifications they could suffer if their space is enlarged such as to include radial excitations too.

In conclusion, a better value of the mass of the Roper resonance has been obtained with the radial excitation $k=1$ of Eq. (10). This result is consistent with the findings of Ref. 13 where a large number of oscillator shells has been taken into account. In addition to being

TABLE $\mathrm{V}$. Results for the strong decay $N+\pi$ and the radiative decay of the first two resonances in the sector $J^{\pi}=\frac{1}{2}^{+}$. First line corresponds to $k=4$ (Refs. 3 and 6), second one to $k=1$ (present results), and the third line is the experiment (Ref. 10). Column 2, the mass (MeV); column 3, the square root of the decay with $\Gamma_{N \pi}^{1 / 2}\left(\mathrm{MeV}^{1 / 2}\right)$; columns 4 and 5, helicity amplitudes $\left(\mathrm{MeV}^{-1 / 2}\right)$ for protons and neutrons.

\begin{tabular}{lcccc}
\hline \hline Resonance & Mass & $\Gamma_{N \pi}^{1 / 2}$ & $A_{1 / 2}^{p}$ & $A_{1 / 2}^{n}$ \\
\hline$P_{11}(1440)$ & 1607 & +12.1 & -31 & +19 \\
& 1485 & +11.5 & -19 & +12 \\
& $1400-1480$ & $10.9_{-3.2}^{+4.8}$ & $-69 \pm 7$ & $+37 \pm 19$ \\
$P_{11}(1710)$ & 1795 & -3.7 & & \\
& 1796 & +2.7 & -48 & +35 \\
& $1680-1740$ & $4.0_{-1.0}^{+1.1}$ & $5 \pm 16$ & -33 \\
\hline
\end{tabular}


efficient in lowering the variational estimate of the Roper-resonance energy, the ansatz (10) provided a rapid way of evaluating decay widths and helicity amplitudes. This is in contrast with a harmonic oscillator or another expansion where problems of slow convergence may arise. A refinement of the quark-photon interaction should be attempted to see whether it could improve the photodecay results.
1J. Carlson, J. Kogut, and V. R. Pandharipande, Phys. Rev. D 27, 233 (1983).

${ }^{2}$ R. Sartor and Fl. Stancu, Phys. Rev. D 31, 128 (1985).

${ }^{3}$ R. Sartor and Fl. Stancu, Phys. Rev. D 33, 727 (1986).

${ }^{4}$ R. Sartor and Fl. Stancu, Phys. Rev. D 34, 3405 (1986).

${ }^{5}$ Fl. Stancu and P. Stassart, Phys. Rev. D 38, 233 (1988).

${ }^{6}$ Fl. Stancu and P. Stassart, Phys. Rev. D 39, 343 (1989).

${ }^{7}$ N. Isgur and G. Karl, Phys. Rev. D 18, 4187 (1978); 19, 2653 (1979); 20, 1191 (1979).

${ }^{8}$ M. Abramowitz and I. A. Stegun, Handbook of Mathematical Functions (Dover, New York, 1970), p. 446.
${ }^{9}$ R. Peierls, Surprizes in Theoretical Physics (Princeton Series in Physics) (Princeton University Press, Princeton, N.J., 1979), p. 61.

${ }^{10}$ Particle Data Group, M. Aguilar-Benitez et al., Phys. Lett. 170B, 1 (1986).

${ }^{11}$ M. B. Gavela, A. Le Yaouanc, L. Oliver, O. Pène, J. C. Raynal, and S. Sood, Phys. Rev. D 21, 182 (1980).

${ }^{12}$ See, e.g., K. Ohta, Phys. Rev. Lett. 43, 1201 (1979), or more recently M. Warns, H. Schröder, W. Pfeil, and H. Rollnick, Bonn University Report No. BONN-ME-89-03 (unpublished). ${ }^{13}$ S. Capstick and N. Isgur, Phys. Rev. D 34, 2809 (1986). 